\title{
Mapping the Morphological Landscape of Oligomeric Di-block Peptide-Polymer Amphiphiles
}

\author{
Benjamin P. Allen, ${ }^{\ddagger[a]}$ Zoe M. Wright, ${ }^{\ddagger[a]}$ Hailey F. Taylor, ${ }^{[a]}$ Thomas J. Oweida, ${ }^{[b]}$ Sabila Kader-Pinky, ${ }^{[b]}$ \\ Emily F. Patteson, ${ }^{[\mathrm{a}]}$ Kara M. Bucci, ${ }^{[\mathrm{a}]}$ Caleb A. Cox,${ }^{[\mathrm{a}]}$ Yaroslava G. Yingling, ${ }^{[\mathrm{b}]}$ and Abigail S. Knight ${ }^{*[\mathrm{a}]}$ \\ [a] B. Allen, Dr. Z. Wright, H. Taylor, E. Patteson, K. Bucci, C. Cox, Prof. A. Knight \\ Department of Chemistry \\ University of North Carolina at Chapel Hill \\ Chapel Hill, North Carolina 27599, United States \\ E-mail: aknight@unc.edu \\ [b] S. Kader-Pinky, Dr. T. Owedia, A. Senthilvel, Prof. Y. Yingling \\ Department of Material Science and Engineering \\ North Carolina State University \\ Raleigh, North Carolina 27695, United States \\ [†] Authors contributed equally
}

\begin{abstract}
Peptide polymer amphiphiles (PPAs) are highly tunable hybrid materials that achieve complex, proteinlike assembly landscapes by combining sequence-dependent properties of peptides with the structural diversity of polymers. Despite their promise as functional biomimetic materials, determining how polymer and peptide properties simultaneously affect PPA self-assembly remains challenging. We herein present a systematic study of critical components within the PPA design space that dictate the self-assembled morphologies. PPAs containing hydrophobic oligo(ethyl acrylate) were used to interrogate the role of polymer molecular weight and dispersity in addition to peptide length and charge density on self-assembly. We observed that PPAs predominantly formed spherical particles (micelles and vesicles), with both polymer molecular weight and peptide hydrophilicity determining morphology. Additionally, peptide charge and polymer dispersity influence particle size. These key benchmarks will facilitate the rational design of PPAs that expand the scope of biomimetic and biocompatible functionality within assembled soft materials.
\end{abstract}

\section{Introduction}

The breadth of tools for controlling the structure and self-assembly of synthetic polymers has recently expanded, bringing synthetic soft materials closer to the catalytic, structural, and templating capabilities of natural proteins. ${ }^{[1-4]}$ Unlike smaller molecules, synthetic polymers have complex, protein-like energetic landscapes that govern their selfassembly, providing the basis for mimicking the morphology-driven functionality of proteins. ${ }^{[5,6]}$ Synthetic polymers can further build upon the abilities of proteins through the range of chemical structures available to non-biological systems via monomer scope and backbone architecture, ${ }^{[7]}$ enabling protein-like behavior in non-biological settings and non-native functions. However, the direct rational design of protein-like polymer assemblies is challenged by our limited ability to determine how multiple inter- and intramolecular interactions will dictate the complex chemical assembly landscapes.

Peptide amphiphiles provide a tunable assembly landscape with which to model relationships between chemical structure, hierarchical self-assembly, and protein-like function. ${ }^{[8]}$ Similar to proteins, peptide amphiphiles frequently use hydrophobic collapse to arrange sequence-defined peptides in space and to control the surrounding chemical environment. $^{[1,4]}$ As peptide sequences can be borrowed directly from natural proteins to create synthetic peptide amphiphiles, biomimetic functionality can be introduced into self-assembled nanostructures with defined morphologies facilitating the study of effects of local environment on peptide activity. ${ }^{[9,10]}$ For example, peptide-lipid conjugates have generated assemblies capable of catalysis, ${ }^{[10,11]}$ metal-binding, ${ }^{[12-14]}$ stimuli-response, ${ }^{[15,16]}$ and cell adhesion ${ }^{[17]}$ while demonstrating the complimentary ways that electrostatics, polarity, hydrogen bonding, and sterics contribute to self-assembly. ${ }^{[18-20]}$ Moving forward, building on this established knowledge base by adding new layers of complexity to the assembly landscape provides opportunities to bring peptide amphiphiles closer to the versatility and functionality of natural proteins.

Peptide-polymer amphiphiles (PPAs), which use a synthetic oligomer tail to drive self-assembly, provide additional tunability to the self-assembly landscape. ${ }^{[21,22]}$ PPAs incorporate both the fine structural control of sequence-defined peptides and the structural diversity of synthetic polymers. The oligomer tail provides tunable properties such as glass 
transition temperature, ${ }^{[23]}$ degree of polymerization (i.e., length), ${ }^{[24]}$ and monomer structure. ${ }^{[25,26]}$ Despite these advantages, it remains challenging to rationally design an oligomer tail to direct self-assembly given a peptide sequence of interest and a target assembly behavior or morphology; predicting the ways in which polymer properties like length dispersity will compete with peptide properties like electrostatics and hydrophilicity is not straightforward. ${ }^{[27],[28]}$ Further, PPA materials exist in a unique design space between peptide-lipid conjugates and block copolymers, where general design rules from both fields may apply but reference points like morphological phase boundaries are not yet established.

To add to the toolbox of design principles for PPAs, we herein present a systematic study of a PPA design space that encompasses both experimental and computational efforts. To establish baseline relationships between hydrophobicity and self-assembly in PPA materials, we selected unstructured peptides that form random coils in solution, minimizing the confounding effects of peptide secondary structure. We experimentally investigated the effects of select polymer properties (molecular weight and dispersity) and peptide properties (length and charge) on self-assembly using transmission electron microscopy (TEM) and image analysis. Computationally, we used molecular dynamics (MD) simulations to quantitatively describe the components of these PPAs. Together these provide key reference points for the design of PPA materials that combine design principles from the parallel fields of block copolymers and peptidelipid conjugates.

\section{Results and Discussion}

\section{Scope of design space and synthesis of PPAs}

To resolve the role of noncovalent interactions that affect the self-assembly of hybrid peptide-polymer amphiphiles (PPAs), we selected a design space that would allow us to independently examine the effects of oligomer tail length and dispersity as well as peptide length and charge on the self-assembled morphology of PPAs. Oligo(ethyl acrylate) was selected for the hydrophobic oligomer tail, as we hypothesized that the short alkyl monomer would allow finetuning of polymer properties. Oligomers were synthesized with average degrees of polymerization $\left(\mathrm{DP}_{\mathrm{n}}\right)$ between 5 and 13 - a molecular weight range spanning that of functional peptide sequences. Atom transfer radical polymerization (ATRP) was implemented to tightly control oligomer length, dispersity, and end group fidelity (Figure 1a and Figures

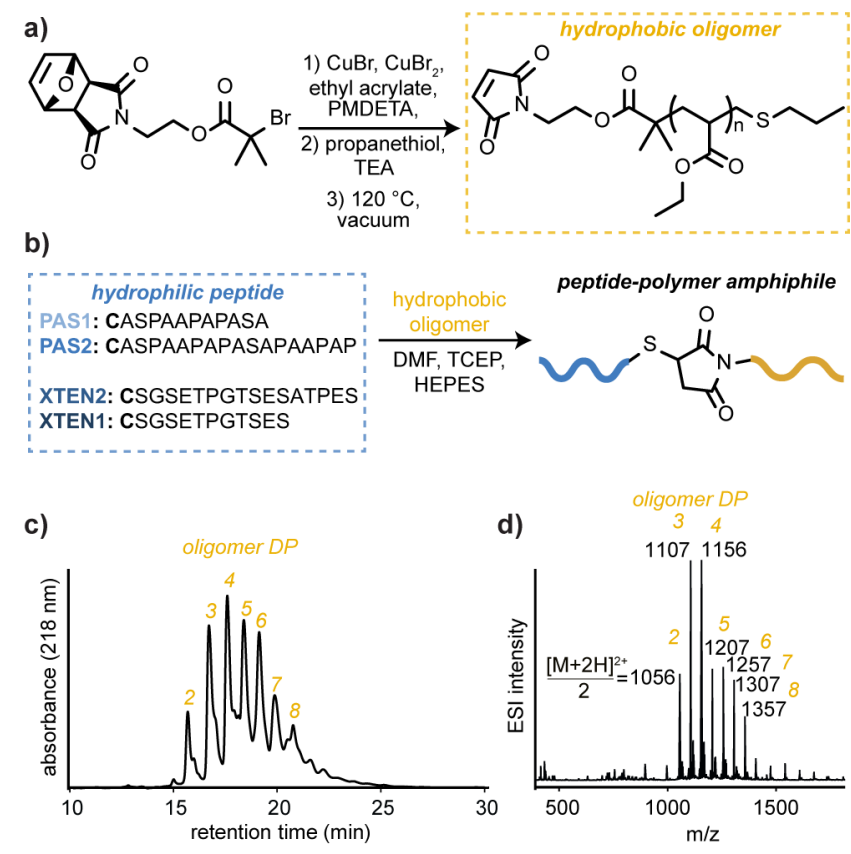

Figure 1. Synthesis of peptide-polymer amphiphiles (PPAs). (a) Maleimideterminated oligomer tails were synthesized by atom transfer radical polymerization (ATRP) followed by substitution of the bromine chain-end and deprotection. (b) PPAs were synthesized through thiol-maleimide coupling, which joins the maleimide-terminated oligomer tail to the $\mathrm{N}$-terminal cysteine of a peptide. (c) The LC chromatogram of a purified PPA shows distinct peaks that correspond to amphiphiles with discrete degrees of polymerization, which (d) can be identified by electrospray ionization (ESI) mass spectrometry. 
S1-3). ${ }^{[29]}$ Post-polymerization substitution of the terminal bromine with propanethiol eliminated any reactivity during further reactions or assembly ${ }^{[30]}$, and a retro-Diels Alder deprotection of the initiator revealed a maleimide allowing facile coupling to a cysteine residue on the peptide ${ }^{[31]}$ (Figures S4-10, Table S1).

Peptide secondary structure has been shown to influence the self-assembly of peptide-containing conjugates; ${ }^{[27,32-35]}$ this is further confounded by the ability of steric crowding to induce secondary structure in peptides. ${ }^{[28,36]}$ Therefore, to focus on the effect of peptide hydrophilicity on self-assembly we used two classes of unstructured peptides $-\mathrm{PAS}^{[37]}$ and XTEN ${ }^{[38-40]}$ - and from these, selected a total of four sequences that vary in length and charge density (Figure 1b, Figures S11-14). Circular dichroism (CD) spectroscopy confirmed that these peptides adopt a random coil conformation in buffer alone ( $\mathrm{pH} 7,10 \mathrm{mM}$ phosphate) and with $20 \%$ trifluoroethanol, which simulates steric crowding $^{[41]}$ (Figures S15-16).

Peptides, synthesized with an N-terminal cysteine, were coupled to a maleimide-terminated oligomer tail to form diblock PPAs via thiol-maleimide coupling, ${ }^{[23]}$ adding in TCEP to ensure the reduction of disulfide bonds (Figure 1b). Characterization of purified PPAs by reversed-phase liquid chromatography-mass spectrometry (LC-MS) showed that each PPA sample contains a collection of amphiphiles with discrete degrees of polymerization (DP), which elute at distinct retention times (Figure 1c) and are identifiable by mass spectrometry (Figure 1d and Figures S17-25). The $\mathrm{DP}_{\mathrm{n}}$ of each batch of PPAs was calculated from the LC spectrum for the purified amphiphiles, as conjugation and purification with a solid-phase extraction column shifted the molecular weight distribution of the PPA slightly relative to the parent polymer sample (Figures S26-27).

\section{PPAs can access a broad range of self-assembled morphologies by adjusting oligomer tail length}

In order to identify the effect of oligomer tail length on PPA morphology, we characterized the self-assembly of a series of PPAs containing the 17-residue charged peptide, XTEN2, and oligo(ethyl acrylate) tails with $\mathrm{DP}_{\mathrm{n}}=5-13$. PPAs $(1 \mathrm{mM})$ were self-assembled by dissolution in HEPES buffer $(\mathrm{pH} 7,50 \mathrm{mM})$ followed by sonication ${ }^{[8]}$ and imaged by both negatively stained and cryogenic transmission electron microscopy (TEM) (Figure 2). Even within this relatively constrained design space, we identified a broad range of self-assembled morphologies that are consistent with the types of morphologies formed by peptide-lipid conjugates and block copolymers: micelles, anisotropic particles (i.e., linear rods and flexible worms), and vesicles (Figure S28). ${ }^{[1,4]}$

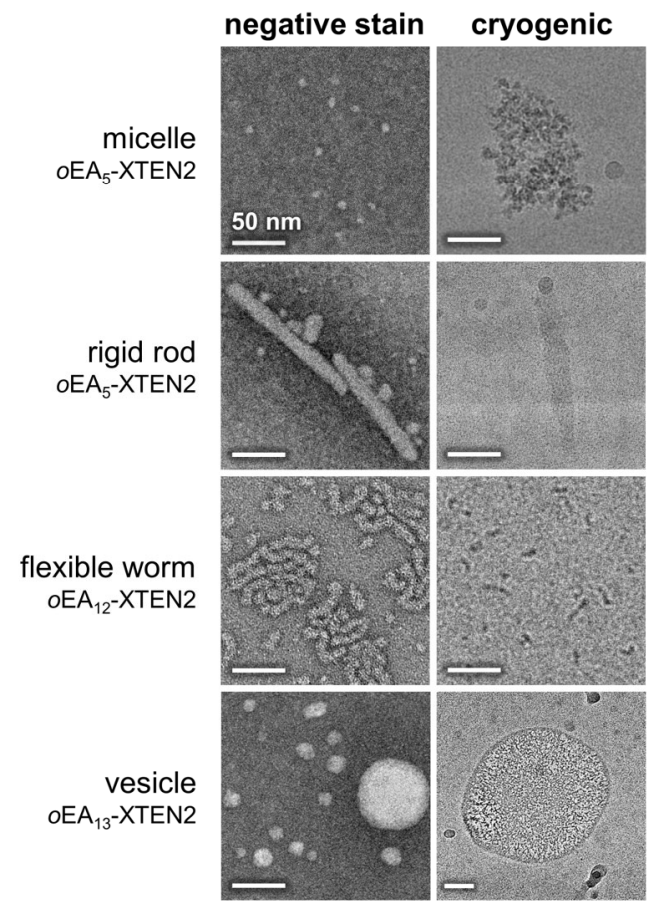

Figure 2. Images from negatively stained (left) and cryogenic (right) transmission electron microscopy (TEM) of major particle types formed by PPA self-assembly. Scale bars represent $50 \mathrm{~nm}$. 
Within this tail length series, we also found that the phase boundaries between morphologies are blurry, with each PPA sample forming simultaneous mixtures of multiple particle types (Figure 3a and Figures S29-34). Specifically, PPAs with shorter oligomer tails (such as $o \mathrm{EA}_{5}$-XTEN2) demonstrate a mixture of smaller spheres (micelles) and linear anisotropic particles (rods). PPAs with longer oligomer tails (such as $o \mathrm{EA}_{13}$-XTEN2) demonstrate a mixture of larger spheres (vesicles) and flexible anisotropic particles (worms). All PPAs, but particularly PPAs with intermediate tail length such as $o \mathrm{EA}_{7}$-XTEN2, contain spherical particles across a range of sizes; cryogenic TEM of $o$ EA ${ }_{6}$ XTEN2 (Figure S35) confirms that micelles and vesicles coexist in our samples.

Remarkably, anisotropic particles represent a minority in the PPAs across this series with the exception of $o \mathrm{EA}_{12^{-}}$ XTEN2, which is at a phase boundary. Most peptide amphiphiles are especially proficient at forming rigid cylindrical micelles, because inter-amphiphile hydrogen bonding provides a strong driving force towards anisotropy. ${ }^{[42-44]}$ In fact, considerable research has been dedicated to modulating this behavior in peptide-lipid conjugates, primarily by altering the peptide sequence to control inter-amphiphile interactions. ${ }^{[4,45-47]}$ This series of XTEN2-based PPAs have a self-assembly space that more closely resembles block copolymers, which typically have a narrow regime for anisotropic particles that are flexible. ${ }^{[48]} \mathrm{We}$ attribute this to the random-coil nature of the XTEN2 peptide.

With multiple morphologies present within a self-assembled PPA, measuring the hydrodynamic diameter using dynamic light scattering (Table S2) resulted in higher average particle sizes than seen in negatively stained TEM images, as has been documented for lipid and gold nanoparticle systems. ${ }^{[49,50]}$ To circumvent this challenge, particle sizes were measured from TEM images to map changes in particle sizes across this series of PPAs (see TEM

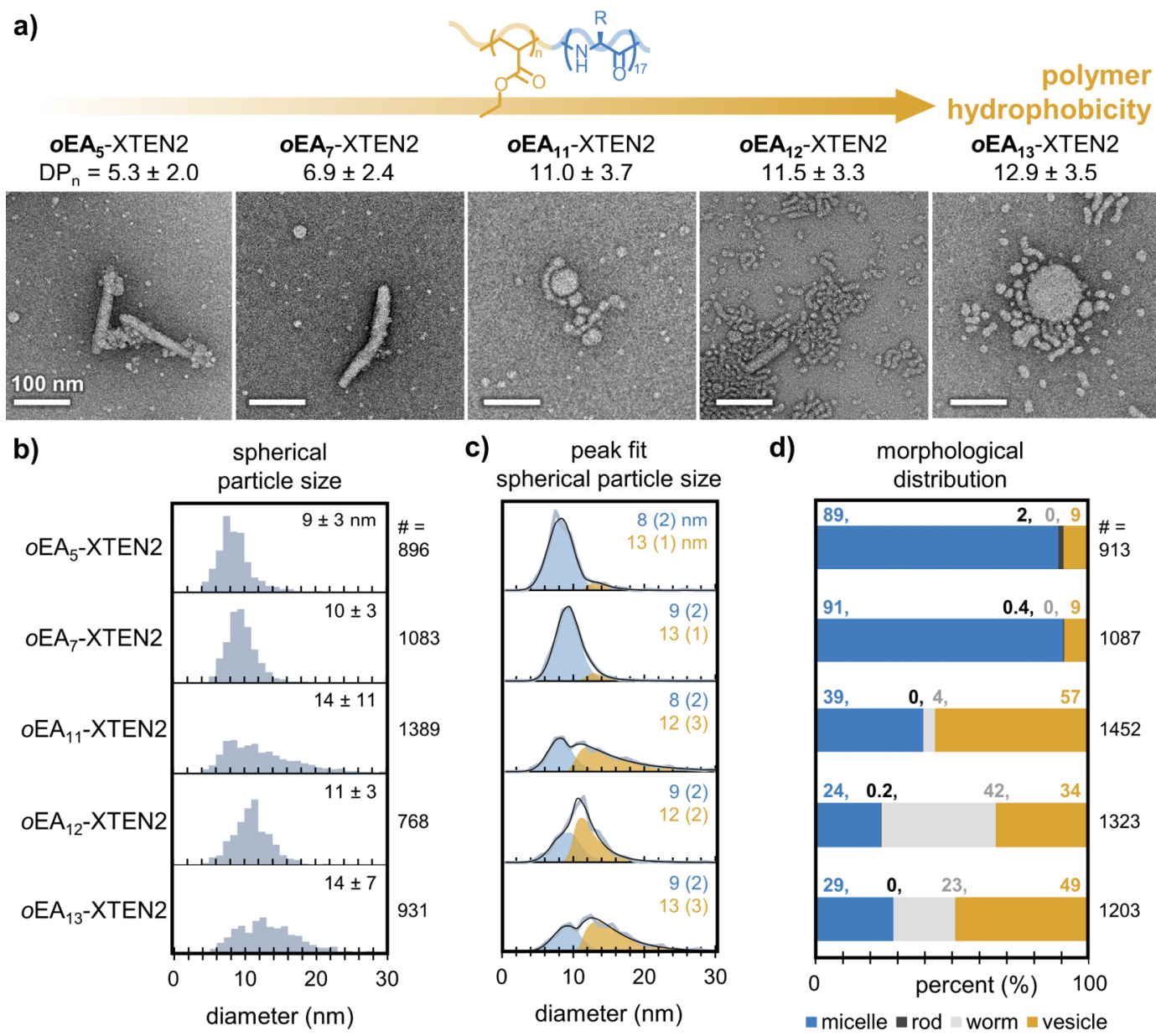

Figure 3. Effect of increasing oligomer tail $\mathrm{DP}_{\mathrm{n}}$ on self-assembly of PPAs with peptide XTEN2. (a) Representative negatively stained TEM images of five PPAs with $\mathrm{DP}_{\mathrm{n}}$ ranging from 5.3 to 12.9; scale bar represents $100 \mathrm{~nm}$. (b) Normalized particle size distributions of spherical particles (excluding anisotropic particles), measured from negatively stained TEM images. Inset values show average diameter \pm standard deviation (to indicate distribution width). Values at right are the total number of spherical particles measured. (c) Peak fitting of spherical particle size distributions: light gray $=$ raw data, blue $=$ Gaussian-fit micelle distribution, yellow = lognormal-fit vesicle distribution, black = sum of blue and yellow curves. Inset text shows peak mode (standard deviation to indicate peak width). (d) Percent of each particle type present in the morphological distribution for each PPA, estimated via peak fitting for the spherical particles. 
quantification supplemental file). As the oligomer tail $\mathrm{DP}_{\mathrm{n}}$ increases, the average diameter of spherical particles increases slightly from 9 to $14 \mathrm{~nm}$ and the particle size distributions become positively skewed due to the increased percentage of vesicles, which typically have a larger size distribution (Figure 3b). ${ }^{[51]}$

To estimate the ratio of micelles to vesicles present in each of our PPA samples, we peak fit the spherical particle size histogram data (Figure 3c) ${ }^{[50]}$ We assumed that each sample would contain some of both spherical particle types, the size distributions for the two types may partially overlap, and that the micelle particle distribution would be Gaussian ${ }^{[52-54]}$ while the vesicle size distribution would be asymmetric and could be reasonably approximated by a lognormal fit. ${ }^{[50,55-58]}$ We were able to consistently identify two peaks for each PPA's spherical particle size distribution with minimal constraints to the fitting parameters, and the sum of these peaks (Figure $3 \mathrm{~b}$, black) demonstrates agreement with the raw data (Figure 3b, light gray). Across this series of PPAs, the Gaussian (micelle) peak was consistently centered between $8-9 \mathrm{~nm}$ and decreased in abundance relative to the lognormal (vesicle) peak, centered between $11-13 \mathrm{~nm}$, as the $\mathrm{DP}_{\mathrm{n}}$ of the PPA sample was increased. This suggests that micelle size remains constant across this series of PPAs.

Using these data to estimate the ratio of micelles to vesicles formed by each PPA, we calculated the percentage of each particle type within their morphological distribution. These morphological distributions clearly show a shift in the particle types present across this series of PPAs (Figure 3d). Short-tailed PPAs form predominantly micelles, while longer-tailed PPAs form predominantly vesicles. Anisotropic particles, manually counted from the original images, show flexible worms are more common with increasing $\mathrm{DP}_{\mathrm{n}}$ while linear rods are most common in the PPA with the shortest tail, $o \mathrm{EA}_{5}$-XTEN2.

Interestingly, the diameter of anisotropic particles appeared to decrease with increasing $\mathrm{DP}_{\mathrm{n}}$ (Figure $\mathrm{S} 36$ ): linear rods observed in short-tailed PPAs $\left(\mathrm{DP}_{\mathrm{n}} 5-12\right)$ have larger diameters on average than the flexible worms observed in longer-tailed PPAs $\left(\mathrm{DP}_{\mathrm{n}} 11-13\right)$. Molecular dynamics simulations of peptide-lipid conjugates have previously shown that as the strength of hydrophobic interactions increases relative to the strength of electrostatic repulsion, peptidelipid assemblies transition from loosely-packed nanofibers with patchy areas of hydrogen bonding in the peptide corona to denser, narrower cylindrical micelles with primarily random coil peptide structure. ${ }^{[59]}$ We thus hypothesized that, in this series of PPAs, hydrogen bonding and hydrophobic interactions together oppose electrostatic repulsion between charged residues in XTEN2 and facilitate the formation of anisotropic particles. In low $\mathrm{DP}_{\mathrm{n}} \mathrm{PPAs}\left(o \mathrm{EA}_{5}\right.$ XTEN2), intermolecular hydrogen bonding is likely necessary for linear rods to remain intact - and, because XTEN2 is primarily a random coil, linear rods are relatively uncommon in the self-assembly space. Long linear fibers are easily identified in cryogenic TEM; these fibers are significantly less prevalent in negatively stained TEM images and may therefore be formed by shear forces in cryogenic TEM grid preparation. ${ }^{[60]}$ We hypothesize that these fibers are similarly built from extended inter-amphiphile hydrogen bonding analogous to those found in amyloid fibers. ${ }^{[61]}$ Finally, in high $\mathrm{DP}_{\mathrm{n}}$ PPAs (e.g., $o \mathrm{EA}_{12}$-XTEN2), hydrophobic interactions are strong enough to enable anisotropic particles to curve without disassembling; these flexible worms do not rely exclusively on inter-amphiphile hydrogen bonding for stability.

\section{Oligomer tail length dispersity contributes to self-assembly behavior}

The mixed morphological phases we observed in this PPA series are likely the result of the length dispersity in the oligomer tails of these amphiphiles. Length dispersity in block copolymer amphiphiles causes the boundaries between self-assembly phases to blur. ${ }^{[4]}$ Disperse samples of block copolymer amphiphiles undergo preferential segregation of amphiphiles with different DPs during self-assembly in order to optimize packing and local curvature; this can lead to the simultaneous formation of multiple particle types, and can subtly affect the size and shape of assembled morphologies ${ }^{[62-65]}$ Notable phase boundary blurriness for block copolymers has been reported at dispersity values of $\mathrm{D} \geq 1.5 ; ;^{[62]}$ however, block copolymer amphiphiles typically range in molecular weight from $20-200 \mathrm{kDa}$, and our PPAs are closer to $2 \mathrm{kDa}$. Thus, despite having dispersity values between $1.00-1.02$, these PPAs have molecular weight distributions and morphological phase boundaries that overlap considerably. We therefore hypothesize that for oligomeric PPAs, individual monomer units create sizable differences in self-assembly behavior.

To experimentally probe the relationship between dispersity and assembled morphology, we created a series of PPAs with $\mathrm{DP}_{\mathrm{n}}=6$ with increasingly narrow molecular weight distributions (MWDs) that eliminated the higher molecular weight tails (Figure 4a, Figure S37-38, see TEM quantification supplemental file). We found that decreasing the dispersity of the oligomer length in the amphiphile resulted in a small shift in the size and polydispersity of the particles formed. The PPA with the broadest MWD formed spherical particles with an average diameter of $12 \mathrm{~nm}$ with $9 \%$ of particles measuring greater than $20 \mathrm{~nm}$ in diameter, while the PPAs with intermediate and narrow MWDs formed spherical particles with average diameters of 11 and $9 \mathrm{~nm}$ respectively with $6 \%$ and $<1 \%$ of particles measuring greater than $20 \mathrm{~nm}$ respectively (Figure $4 \mathrm{~b}$ ). Results of the peak fitting suggest that the particle diameter of both 
micelle and vesicle populations are affected (Figure 4c). This is consistent with block copolymer assembly within a vesicle-forming regime, where amphiphiles with shorter solvophobic blocks form smaller vesicles. ${ }^{[63,65]}$

Notably, the $o \mathrm{EA}_{6}$-XTEN2 materials characterized in Figure 4 were each synthesized with materials from different polymer syntheses. Fractionation was used during purification with a reversed-phase solid-phase extraction column to independently control $\mathrm{DP}_{\mathrm{n}}$ and dispersity. Despite using different starting materials, these amphiphiles demonstrate similar morphological distributions (Figure 4d), suggesting a strong correlation between amphiphile $\mathrm{DP}_{\mathrm{n}}$ and the mixture of particle types formed. Further, the phase boundary between micelles and vesicles for $o \mathrm{EA}_{\mathrm{n}}$-XTEN2 materials likely resides in the range of DP $=4-7$ as these are the only DPs shared between all three materials.
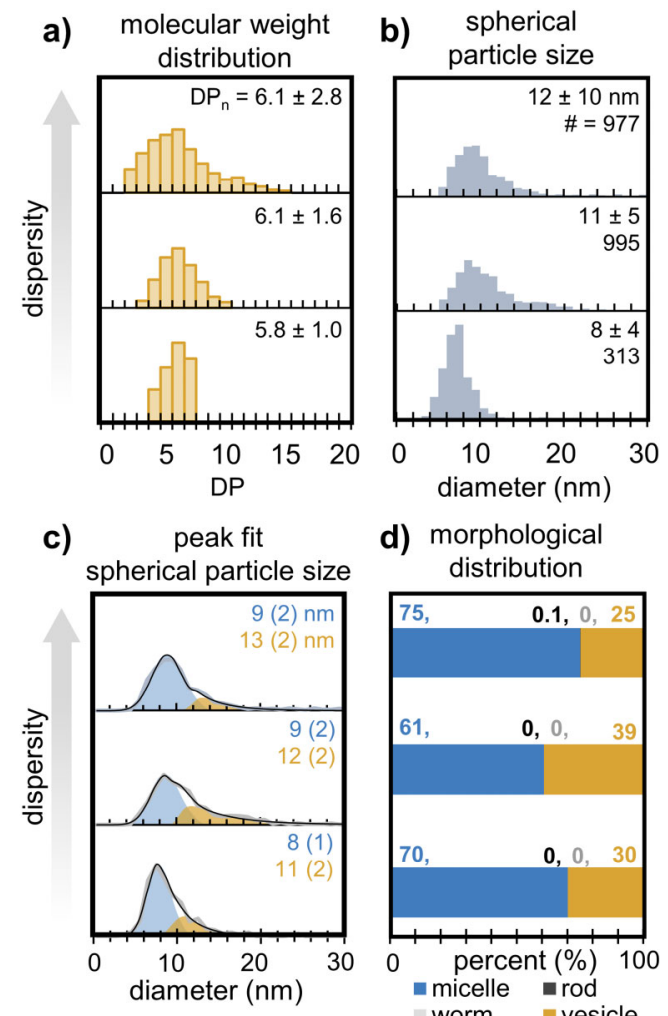

d) morphological

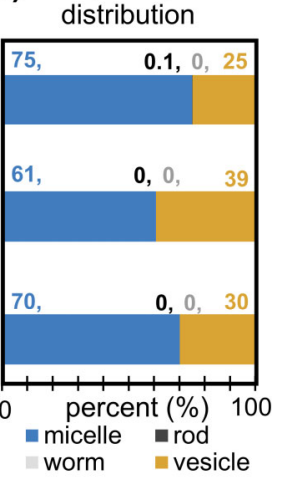

Figure 4. Assembly behavior of PPAs with the same average $D P_{n}$ but different tail length dispersities ranging from broadest (top of each panel) to narrowest (bottom). (a) Molecular weight distributions. (b) Spherical particle size distributions. Inset text shows average particle diameter \pm standard deviation and total number of particles measured. (c) Peak fitting for spherical particle size distributions; light gray = raw data, blue $=$ Gaussian fit micelle distribution, yellow = lognormal fit vesicle distribution, black = sum of blue and yellow curves. Inset text shows peak mode (standard deviation indicating peak width). (d) Percent of each particle type present in the morphological distribution for each PPA, estimated via peak fitting for spherical particles.

\section{Peptide hydrophilicity modulates self-assembly and determines the limits of accessible morphologies}

PPAs are of particular interest to the field of protein-mimetic materials because of their potential to directly incorporate peptides with documented biological functionality while recapitulating basic features of protein-like hydrophobic collapse. ${ }^{[68]}$ However, designing a PPA with a particular self-assembled morphology from a defined peptide sequence places unique constraints on the tools that can be used to control assembly, and requires an understanding of how the target peptide sequence influences or limits the assembly behavior.

To characterize the effect of peptide hydrophilicity on assembly, we first characterized the differences in hydrophilicity between our four selected random-coil peptides, which vary in length and number of charged residues: XTEN1 (12 residues with 1 positive and 2 negative charges) and XTEN2 (17 residues with 1 positive and 3 negative charges) are charged at $\mathrm{pH} 7$, while PAS1 (12 residues) and PAS2 (18 residues) are neutral except for a positive charge on the N-terminus. Hansen solubility parameter (HSP) values ${ }^{[66,67]}$, calculated for each peptide using molecular 
dynamics (MD) simulations, suggest that the most hydrophilic peptide is XTEN1 followed by XTEN2, PAS1, and PAS2 (Figure S39a); however, while this calculation captures the effects of charge on hydrophilicity, it does not account for the effect of hydrogen bonding with water and thus underestimates the hydrophilicity of the PAS peptides $^{[37]}$. By simulating hydrogen bonding of each peptide within a solvated environment using $\mathrm{MD}^{[69]}$, we found that the capacity for hydrogen bonding between the peptide and water varies significantly with sequence and length (Figure S39b). We experimentally corroborated these calculations by comparing HPLC retention time of the peptides in a neutral solvent system that matched the $\mathrm{pH}$ of our assembly conditions (Figure $\mathrm{S} 40$ ). The retention times suggest the peptides follow this order in decreasing hydrophilicity: XTEN1, XTEN2, PAS2, PAS1 - with PAS1 being considerably less hydrophilic than PAS2.

Each of these random-coil peptides was conjugated to oligo(ethyl acrylate) $\mathrm{DP}_{\mathrm{n}}=5$, as we hypothesized that this would result in a range of self-assembled morphologies with minimal amphiphile insolubility. Similar to the previous oligomer tail series (Figure 3), PPAs across this peptide series formed primarily spherical particles of various sizes, with anisotropic particles representing a minority of particles (Figure 5a, Figures S41-45, see TEM quantification supplemental file). The random coil structure of the peptides across this series likely contributes to the higher fraction of spherical, rather than anisotropic, particles. Though the limited electrostatic repulsion in the less charged PAS peptides might be expected to facilitate intermolecular hydrogen bonding (and thus formation of anisotropic particles) compared to the charged XTEN2, PAS in particular derives its random-coil structure from the proline residues distributed irregularly throughout its sequence. These proline residues introduce kinks in the peptide backbone that force the peptide to resist hydrophobic collapse and may also interfere with the formation of inter-amphiphile hydrogen bonds.
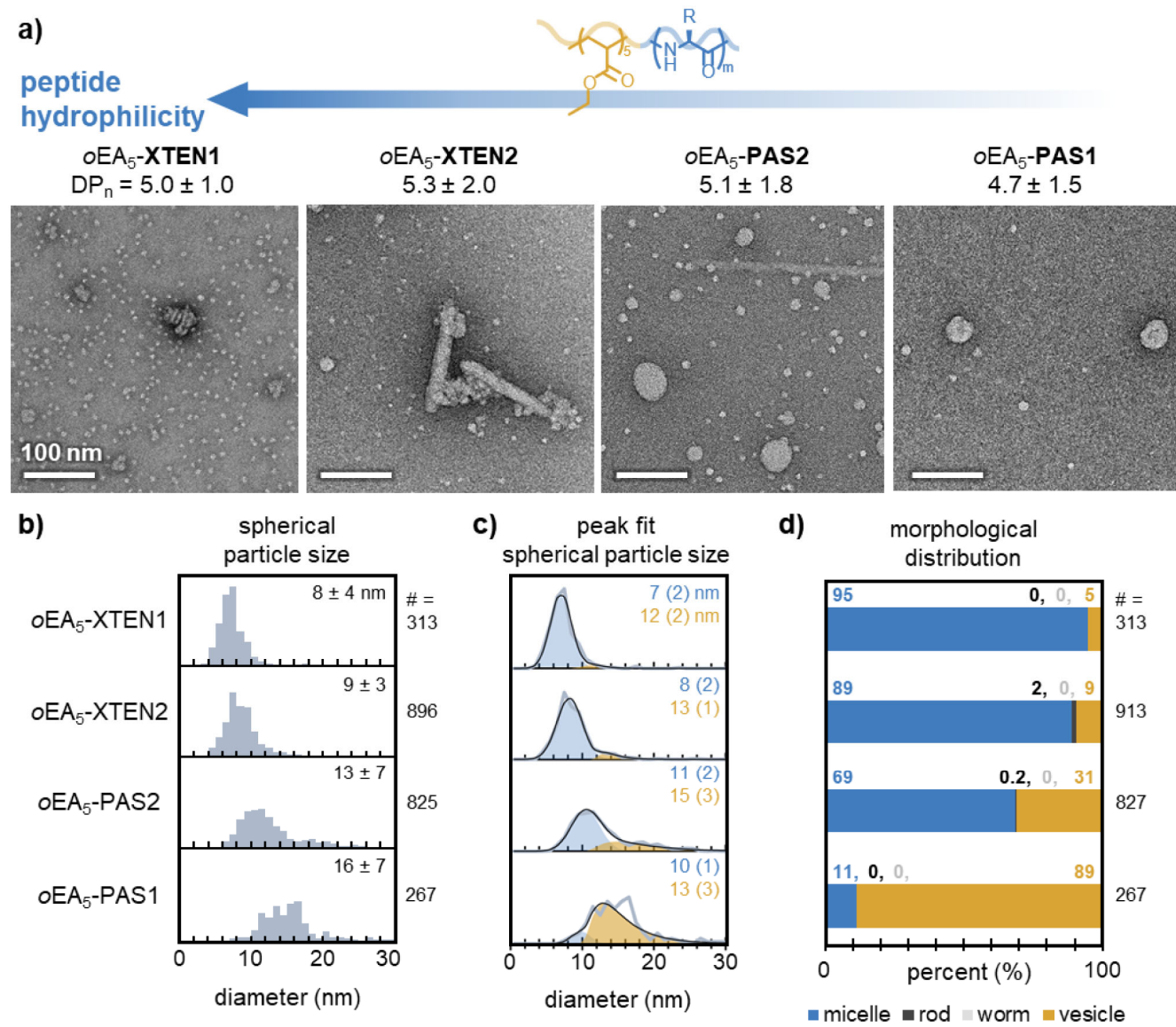

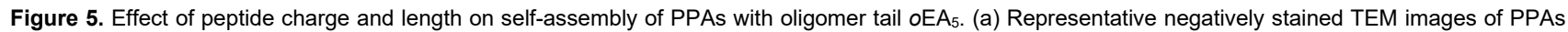
containing each of the selected peptide sequences; scale bar represents $100 \mathrm{~nm}$. (b) Normalized particle size distributions of spherical particles (excluding anisotropic particles), measured from negatively stained TEM images. Inset values show average diameter \pm standard deviation. Values at right are the total number of spherical particles measured. (c) Peak fitting of spherical particle size distributions: light gray $=$ raw data, blue $=$ Gaussian-fit micelle distribution, yellow = lognormal-fit vesicle distribution, black = sum of blue and yellow curves. Inset text shows peak mode (standard deviation indicating peak width). (d) Percent of each particle type present in the morphological distribution for each PPA, estimated via peak fitting for spherical particles. 
Notably, $o \mathrm{EA}_{5}$-XTEN1 failed to form well-defined particles under the previous assembly conditions (Figure S43) and only formed discrete particles upon the addition of $100 \mathrm{mM} \mathrm{NaCl}$ to the assembly buffer (Figure 5a, Figure S44). The high charge density of XTEN1 is likely responsible for the failure of $o \mathrm{EA}_{5}$-XTEN1 to self-assemble without $\mathrm{NaCl}$, outcompeting the hydrophobic interactions provided by the $\mathrm{DP}_{\mathrm{n}}=5$ oligo(ethyl acrylate) tail. To ensure the added $\mathrm{NaCl}$ did not have a significant effect on the morphological distribution, $o \mathrm{EA}_{5}-\mathrm{XTEN} 2$ was assembled with and without $\mathrm{NaCl}$ and comparable ratios of morphologies were observed (Figure S45-46).

With decreasing peptide hydrophilicity, we found an increase in the average spherical particle size across the series of PPAs, from $8 \mathrm{~nm}$ in $o \mathrm{EA}_{5}$-XTEN1 (with NaCl) to $16 \mathrm{~nm}$ in $o \mathrm{EA}_{5}$-PAS1 (Figure 5b). Results from peak fitting of the spherical particle size distribution suggests that PPAs with charged peptides XTEN1 and XTEN2 form slightly smaller micelles on average than the less charged PAS1 and PAS2 (Figure 5c). This is consistent with peptide-lipid conjugates: highly charged conjugates form smaller micelles than neutral conjugates, minimizing electrostatic interactions between charged amphiphiles by maximizing the curvature of the micelle assembly. ${ }^{[19]}$ Additionally, assembly of $o \mathrm{EA}_{5}$-XTEN2 (17 amino acids) in the presence of $100 \mathrm{mM} \mathrm{NaCl}$ produced the same ratio of micelles to vesicles as $o \mathrm{EA}_{5}$-PAS2 (18 amino acids) in the absence of $\mathrm{NaCl}$ and the micelles were similar in size (Figure S46b, see TEM quantification supplemental file).

Further, we found that the morphological distributions across this series of PPAs show a clear shift from heavily micelle-dominated in $o \mathrm{EA}_{5}$-XTEN1 to increasingly vesicle-dominated with decreasing peptide hydrophilicity (Figure $5 \mathrm{~d})$. Notably, the ratio of micelles to vesicles present in the PPA materials across this series, determined by peak fitting, shows a strong, significant relationship with the relative retention time of the free peptides on HPLC at pH 7. Plotting the log of the ratio of micelles to vesicles against retention time yields a linear regression with an $\mathrm{R}^{2}$ value of 0.95 and a $p$ value of 0.02 (Figure $\mathrm{S} 40 \mathrm{~b}$ ). This suggests that, for these materials, the retention time of unstructured peptides by HPLC at a $\mathrm{pH}$ relevant to the assembly can be a predictive tool that captures the combination of solubility (e.g., HSP values) and water interactions (e.g., hydrogen bonds with water) that contribute to self-assembly behavior.

To further probe the limits of tunability in this PPA system, a sample of $o \mathrm{EA}_{5}$-PAS1 was fractionated during purification such that only the smallest DP (least hydrophobic) amphiphiles were collected, yielding a sample with
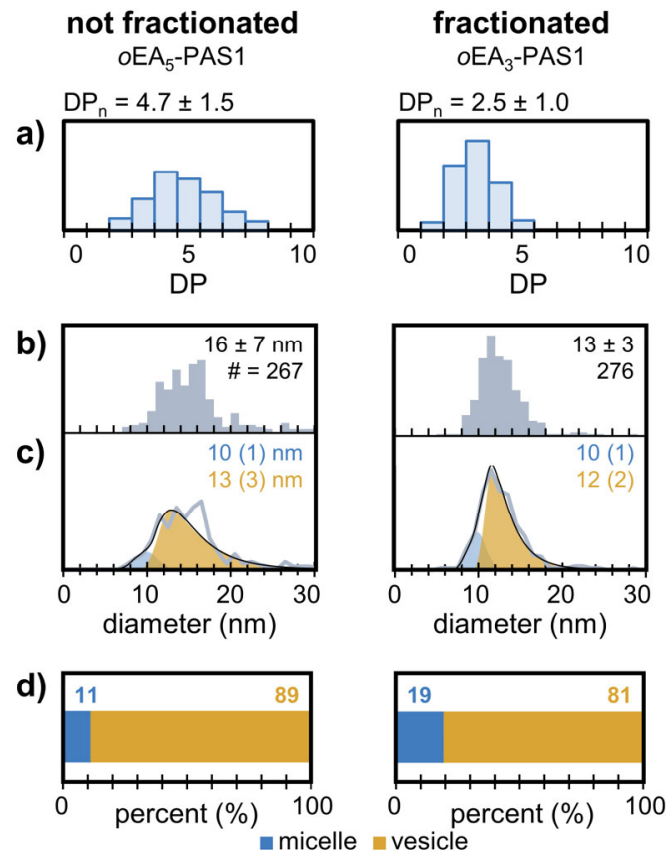

Figure 6. Fractionation of $o E A_{5}-P A S 1$ during purification yields a PPA sample with (a) a narrower molecular weight distribution and $\mathrm{DP}_{\mathrm{n}}$ of $2.5 \pm$ 1.0. (b) Normalized particle size distributions of spherical particles. Inset text shows average diameter \pm standard deviation. (c) Peak fitting scheme; light gray $=$ raw data, blue $=$ Gaussian fit micelle distribution, yellow $=$ lognormal fit vesicle distribution, black = sum of blue and yellow curves. Inset text shows peak mode (standard deviation, indicating peak width). (d) Percent of each particle type present in the morphological distribution for each PPA, estimated via peak fitting. 
$\mathrm{DP}_{\mathrm{n}}=2.5$ (Figure $6 \mathrm{a} ; o \mathrm{EA}_{3}-\mathrm{PAS} 1$ ). When assembled, $o \mathrm{EA}_{3}-\mathrm{PAS} 1$ formed exclusively spheres with no anisotropic particles, analogous to the unfractionated $o \mathrm{EA}_{5}$-PAS1 (Figure S47). However, the spheres formed by $o \mathrm{EA}_{3}-\mathrm{PAS} 1$ were smaller on average than those formed by $o \mathrm{EA}_{5}$-PAS1 (Figure 6b): $o \mathrm{EA}_{3}-\mathrm{PAS} 1$ particles had an average diameter of $13 \mathrm{~nm}$ compared to $o \mathrm{EA}_{5}$-PAS1 particles with an average diameter of $16 \mathrm{~nm}$ (see TEM quantification supplemental file). Further, the results of the peak fitting (Figure 6c) show that reducing the $\mathrm{DP}_{\mathrm{n}}$ of the oligomer tail results in only a small increase in the percentage of micelles (Figure 6d). This is in direct contrast to the trend observed in hydrophilic XTEN2 PPAs (Figure 3) and suggests that the phase boundary for the formation of micelles in $o \mathrm{EA}_{\mathrm{n}}$-PAS1 PPAs is beyond what is physically accessible - to form micelles with PAS1, a different oligomer tail and/or a modified PAS1 sequence with additional hydrophilic residues would need to be used.

\section{Conclusion}

Peptide-polymer amphiphiles (PPAs) are promising hybrid materials that merge protein-mimetic functionality with the synthetic versatility of block copolymers. Though these materials obey the general rules of self-assembly driven by hydrophobic collapse, our understanding of how structural features individually contribute to self-assembly behavior is not yet comprehensive enough to enable predictive capabilities. Here, we contribute a systematic study of how the structural features of PPAs - oligomer tail length and dispersity, and peptide charge and length - can be tuned to impact aqueous self-assembly. Within a series of short oligomeric tails, we demonstrate that PPAs can span the entire morphological regime of micelles, anisotropic particles, and vesicles. We also show that the morphological distribution of a PPA can be engineered by adjusting the length of the oligomer tail and is impacted by the hydrophilicity of the selected peptide. Additionally, the average particle size can be tuned through the length dispersity of the oligomeric tail and/or the charge of the peptide. By pairing these experimental efforts with computational modeling to quantitatively describe the hydrophobicity of our amphiphile components, we have established important benchmarks that will facilitate the rational design of sophisticated and well-controlled PPA assemblies.

Future work with PPAs will continue to bring together the structural diversity afforded by polymers and the complexity of interactions afforded by peptides to open new possibilities in soft materials. Additional structural variations in the oligomer tail could potentially be exploited to tune the stability, dynamics, and responsiveness to stimuli (e.g., temperature and $\mathrm{pH}$ ) of PPA assemblies. An enhanced understanding of how polymer properties correlate with the properties of hierarchical assemblies will enable synthetic materials to mimic and expand upon the specific binding and catalytic capabilities of proteins.

\section{Acknowledgements}

This material is based upon experimental work (A.S.K.) supported by the Air Force Office of Scientific Research under Award Number FA9550-20-1-0172 and computational work (Y.G.Y.) supported through the National Science Foundation Macromolecular, Supramolecular, and Nanochemistry Program under Award Number CHE-2108111. B.P.A. thanks the UNC Program in Molecular and Cellular Biophysics (MCBP) National Institutes of Health (NIH) training fellowship (T32GM008570). TEM was performed in part at the Chapel Hill Analytical and Nanofabrication Laboratory, CHANL, a member of the North Carolina Research Triangle Nanotechnology Network, RTNN, which is supported by the National Science Foundation, Grant ECCS-1542015, as part of the National Nanotechnology Coordinated Infrastructure, NNCI. We acknowledge Jared Peck and Dr. Joshua Strauss of the UNC CryoTEM Core Facility for technical assistance in this project. We would also like to acknowledge the Leibfarth (GPC), Dick (DLS, Nanopure water), and Johnson (dry solvents) laboratories for assistance with instrumentation and solvents. Additionally, we acknowledge the University of North Carolina's Department of Chemistry NMR Core Laboratory.

Keywords: peptide-polymer amphiphile, self-assembly, electron microscopy, biomimetic, supramolecular chemistry

[1] M. H. Barbee, Z. M. Wright, B. P. Allen, H. F. Taylor, E. F. Patteson, A. S. Knight, Macromolecules 2021, $54,3585-3612$

[2] Y. Mai, A. Eisenberg, Chem. Soc. Rev. 2012, 41, 5969-5985.

[3] E. Krieg, M. M. C. Bastings, P. Besenius, B. Rybtchinski, Chem. Rev. 2016, 116, 2414-2477.

[4] C. Chen, R. A. L. L. Wylie, D. Klinger, L. A. Connal, Chem. Mater. 2017, 29, 1918-1945.

[5] T. Nicolai, O. Colombani, C. Chassenieux, Soft Matter 2010, 6, 3111-3118.

[6] K. A. Rubinson, Protein J 2019, 38, 95-119 
[7] A. Blanazs, S. P. Armes, A. J. Ryan, Macromol. Rapid Commun. 2009, 30, 267-277.

[8] A. Dasgupta, D. Das, Langmuir 2019, 35, 10704-10724.

[9] J. H. Ortony, C. J. Newcomb, J. B. Matson, L. C. Palmer, P. E. Doan, B. M. Hoffman, S. I. Stupp, Nat. Mater. 2014, 13, 812-816.

[10] S. B. P. E. Timmermans, J. C. M. van Hest, Curr. Opin. Colloid Interface Sci. 2018, 35, 26-35.

[11] O. Zozulia, M. A. Dolan, I. V. Korendovych, Chem. Soc. Rev. 2018, 47, 3621-3639.

[12] S. Gui, Y. Huang, Y. Zhu, Y. Jin, R. Zhao, ACS Appl. Mater. Interfaces 2019, 11, 5804-5811.

[13] P. Sharma, H. Kaur, S. Roy, Biomacromolecules 2019, 20, 2610-2624.

[14] R. Singh, N. K. Mishra, N. Singh, P. Rawal, P. Gupta, K. B. Joshi, New J. Chem. 2020, 44, 9255-9263.

[15] G. Zaldivar, S. Vemulapalli, V. Udumula, M. Conda-Sheridan, M. Tagliazucchi, J. Phys. Chem. C 2019, 123, 17606-17615.

[16] C. Gao, H. Li, Y. Li, S. Kewalramani, L. C. Palmer, V. P. Dravid, S. I. Stupp, M. Olvera De La Cruz, M. J. Bedzyk, J. Phys. Chem. B 2017, 121, 1623-1628.

[17] M. J. Webber, J. Tongers, M.-A. Renault, J. G. Roncalli, D. W. Losordo, S. I. Stupp, Acta Biomater. 2010, 6, 3-11.

[18] H. Cui, M. J. Webber, S. I. Stupp, Pept. Sci. 2010, 94, 1-18.

[19] G. Zaldivar, S. Vemulapalli, V. Udumula, M. Conda-Sheridan, M. Tagliazucchi, J. Phys. Chem. C 2019, 123, 17606-17615.

[20] M. P. Hendricks, K. Sato, L. C. Palmer, S. I. Stupp, Acc. Chem. Res. 2017, 50, 2440-2448.

[21] J. Y. Shu, B. Panganiban, T. Xu, Annu. Rev. Phys. Chem. 2013, 64, 631-657.

[22] L. M. Randolph, M. P. Chien, N. C. Gianneschi, Chem. Sci. 2012, 3, 1363-1380.

[23] A. S. Knight, J. Larsson, J. M. Ren, R. Bou Zerdan, S. Seguin, R. Vrahas, J. Liu, G. Ren, C. J. Hawker, J. Am. Chem. Soc. 2018, 140, 1409-1414.

[24] R. Otter, P. Besenius, Org. Biomol. Chem. 2019, 17, 6719-6734.

[25] D. B. Wright, A. Ramírez-Hernández, M. A. Touve, A. S. Carlini, M. P. Thompson, J. P. Patterson, J. J. De Pablo, N. C. Gianneschi, ACS Macro Lett. 2019, 8, 676-681.

[26] A. Saha, S. Jana, T. K. Mandal, J. Polym. Sci. Part A Polym. Chem. 2016, 54, 3019-3031.

[27] M. M. J. Van Rijt, A. Ciaffoni, A. Ianiro, M. A. Moradi, A. L. Boyle, A. Kros, H. Friedrich, N. A. J. M. Sommerdijk, J. P. Patterson, Chem. Sci. 2019, 10, 9001-9008.

[28] F. Versluis, H. R. Marsden, A. Kros, Chem. Soc. Rev. 2010, 39, 3434-3444.

[29] K. Matyjaszewski, Macromolecules 2012, 45, 4015-4039.

[30] A. H. Soeriyadi, C. Boyer, F. Nyström, P. B. Zetterlund, M. R. Whittaker, J. Am. Chem. Soc. 2011, 133, 11128-11131.

[31] E. H. Discekici, A. H. St. Amant, S. N. Nguyen, I.-H. Lee, C. J. Hawker, J. Read de Alaniz, J. Am. Chem. Soc. 2018, 140, 5009-5013.

[32] C. A. Machado, I. R. Smith, D. A. Savin, Macromolecules 2019, 52, 1899-1911.

[33] J. Li, X. Du, S. Hashim, A. Shy, B. Xu, J. Am. Chem. Soc. 2017, 139, 71-74.

[34] T. Shimada, S. Lee, F. S. Bates, A. Hotta, M. Tirrell, J. Phys. Chem. B 2009, 113, 13711-13714.

[35] D. Missirlis, A. Chworos, C. J. Fu, H. A. Khant, D. V. Krogstad, M. Tirrell, Langmuir 2011, 27, 6163-6170.

[36] D. W. P. M. Löwik, J. Garcia-Hartjes, J. T. Meijer, J. C. M. Van Hest, Langmuir 2005, 21, 524-526.

[37] J. Breibeck, A. Skerra, Biopolymers 2018, 109, 1-12.

[38] V. Schellenberger, C. W. Wang, N. C. Geething, B. J. Spink, A. Campbell, W. To, M. D. Scholle, Y. Yin, Y. Yao, O. Bogin, J. L. Cleland, J. Silverman, W. P. C. Stemmer, Nat. Biotechnol. 2009, 27, 1186-1190.

[39] A. C. Komor, Y. B. Kim, M. S. Packer, J. A. Zuris, D. R. Liu, Nature 2016, 533, 420-424.

[40] V. N. Podust, S. Balan, B. C. Sim, M. P. Coyle, U. Ernst, R. T. Peters, V. Schellenberger, J. Control. Release 2016, 240, 52-66.

[41] F. D. Sonnichsen, J. E. Van Eyk, R. S. Hodges, B. D. Sykes, Biochemistry 1992, 31, 8790-8798.

[42] S. E. Paramonov, H. Jun, J. D. Hartgerink, J. Am. Chem. Soc., 2006, 128, 7291-7298.

[43] Z. Hua, J. R. Jones, M. Thomas, M. C. Arno, A. Souslov, T. R. Wilks, R. K. O’Reilly, Nat. Commun. 2019, $10,1-8$.

[44] J. D. Hartgerink, E. R. Zubarev, S. I. Stupp, Curr. Opin. Solid State Mater. Sci. 2001, 5, 355-361.

[45] Z. Yu, F. Tantakitti, L. C. Palmer, S. I. Stupp, Nano Lett. 2016, 16, 6967-6974.

[46] H. Cui, T. Muraoka, A. G. Cheetham, S. I. Stupp, Nano Lett. 2009, 9, 945-951.

[47] H. Cui, A. G. Cheetham, E. T. Pashuck, S. I. Stupp, J. Am. Chem. Soc. 2014, 136, 12461-12468. 
[48] B. Zhang, X. Lv, A. Zhu, J. Zheng, Y. Yang, Z. An, Macromolecules 2018, 51, 2776-2784.

[49] J. Pencer, F. R. Hallett, Langmuir 2003, 19, 7488-7497.

[50] B. Lee, S. Yoon, J. W. Lee, Y. Kim, J. Chang, J. Yun, J. C. Ro, J. S. Lee, J. H. Lee, ACS Nano 2020, 14, 17125-17133.

[51] M. Winterhalter, D. D. Lasic, Chem. Phys. Lipids 1993, 64, 35-43.

[52] P. H. Nelson, G. C. Rutledge, T. A. Hatton, J. Chem. Phys. 1997, 107, 10777-10781.

[53] A. L. R. Bug, M. E. Cates, S. A. Safran, T. A. Witten, J. Chem. Phys. 1987, 87, 1824-1833.

[54] M. Nakano, M. Deguchi, K. Matsumoto, H. Matsuoka, H. Yamaöka, Macromolecules 1999, 32, 7437-7443.

[55] F. Chécot, A. Brûlet, J. Oberdisse, Y. Gnanou, O. Mondain-Monval, S. Lecommandoux, Langmuir 2005, 21, 4308-4315.

[56] R. Sigel, M. Łosik, H. Schlaad, Langmuir 2007, 23, 7196-7199.

[57] B. A. Korgel, J. H. Van Zanten, H. G. Monbouquette, Biophys. J. 1998, 74, 3264-3272.

[58] N. W. Song, K. M. Park, I. H. Lee, H. Huh, Metrologia 2009, 46, 480-488.

[59] I. W. Fu, C. B. Markegard, B. K. Chu, H. D. Nguyen, Langmuir 2014, 30, 7745-7754.

[60] B. F. B. Silva, E. F. Marques, U. Olsson, Langmuir 2008, 24, 10746-10754.

[61] Y. S. Velichko, S. I. Stupp, M. O. de la Cruz, J. Phys. Chem. B 2008, 112, 2326-2334.

[62] K. E. B. Doncom, L. D. Blackman, D. B. Wright, M. I. Gibson, R. K. O'Reilly, Chem. Soc. Rev. 2017, 46, 4119-4134.

[63] A. L. Schmitt, M. H. Repollet-Pedrosa, M. K. Mahanthappa, ACS Macro Lett. 2012, 1, 300-304.

[64] Y. Jiang, T. Chen, F. Ye, H. Liang, A. C. Shi, Macromolecules 2005, 38, 6710-6717.

[65] X. Li, P. Tang, F. Qiu, H. Zhang, Y. Yang, J. Phys. Chem. B 2006, 110, 2024-2030.

[66] M. Belmares, M. Blanco, W. A. Goddard III, R. B. Ross, G. Caldwell, S. -H. Chou, J. Pham, P. M. Olofson, C. Thomas, J. Comput. Chem. 2004, 25, 1814-1826.

[67] L. Martínez, R. Andrade, E. G. Birgin, J. M. Martínez, J. Comput. Chem. 2009, 30, 2157-2164.

[68] E. Kokkoli, A. Mardilovich, A. Wedekind, E. L. Rexeisen, A. Garg, J. A. Craig, Soft Matter 2006, 2, 10151024.

[69] N. G. Taylor, S. H. Chung, A. L. Kwansa, R. R. Johnson III, A. J. Teator, N. J. B. Milliken, K. M. Koshlap, Y. G. Yingling, Y. Z. Lee, F. A. Leibfarth, Chem. Eur. J. 2020, 26, 9982-9990. 